\title{
Review and Designs of Federated Management in Future Internet Architectures
}

\author{
Martín Serrano ${ }^{1}$, Steven Davy ${ }^{1}$, Martin Johnsson ${ }^{1}$, Willie Donnelly ${ }^{1}$, and Alex Galis ${ }^{2}$ \\ ${ }^{1}$ Waterford Institute of Technology - WIT \\ Telecommunications Software and Systems Group - TSSG, Co. Waterford, Ireland \\ \{jmserrano, sdavy, mjohnsson, wdonnelly\}atssg.org \\ ${ }^{2}$ University College London - UCL \\ Department of Electronic and Electrical Engineering, Torrington Place, London, U.K. \\ a.galis@ee.ucl.ac.uk
}

\begin{abstract}
The Future Internet as a design conception is network and serviceaware addressing social and economic trends in a service oriented way. In the Future Internet, applications transcend disciplinary and technology boundaries following interoperable reference model(s). In this paper we discuss issues about federated management targeting information sharing capabilities for heterogeneous infrastructure. In Future Internet architectures, service and network requirements act as design inputs particularly on information interoperability and cross-domain information sharing. An inter-operable, extensible, reusable and manageable new Internet reference model is critical for Future Internet realisation and deployment. The reference model must rely on the fact that highlevel applications make use of diverse infrastructure representations and not use of resources directly. So when resources are not being required to support or deploy services they can be used in other tasks or services. As implementation challenge for controlling and harmonising these entire resource management requirements, the federation paradigm emerges as a tentative approach and potentially optimal solution. We address challenges for a future Internet Architecture perspective using federation. We also provide, in a form of realistic implementations, research results and solutions addressing rationale for federation, all this activities are developed under the umbrella of federated management activity in the Future Internet.
\end{abstract}

Keywords: Federation, Management, Reference Model, Future Internet, Architectures and Systems, Autonomics, Service Management, Semantic Modelling and Management, Knowledge Engineering, Networking Data and Ontologies, Future Communications and Internet.

\section{Introduction}

In recent years convergence on Internet technologies for communication's, computation's and storage's networks and services has been a clear trend in the Information and Communications Technology (ICT) domain. Although widely discussed and 
researched, this trend has not fully run its course in terms of implementation, due to many complex issues involving deployment of non-interoperable and management infrastructural aspects and also due to technological, social, economic restrictions and bottlenecks in the Future Internet.

In the Future Internet, services and networks follow a common goal: to provide solutions in a form of implemented interoperable mechanisms. Telecommunications networks have undergone a radical shift from a traditional circuit-switched environment with heavy/complex signalling focused on applications-oriented perspective, towards a converged service-oriented space, mostly Internet interaction by customer as end-user and network operators as service providers. The benefits of this shift reflect cost reduction and increase systems flexibility to react to user demands, by replacing a plethora of proprietary hardware and software platforms with generic solutions supporting standardised development and deployment stacks.

The Future Internet as design conception is service-aware of the network infrastructure addressing service-oriented, social trends and economic commitments. In the Future Internet trans-disciplinary solutions (applications that transcend disciplinary boundaries) following reference model(s) are crucial for a realistic integrated management realisation. Challenges in the future communications systems mainly demand, in terms of end user requirements, personalized provisioning, service-oriented performance, and service-awareness networking.

Additionally to those technology requirements, necessities to support information interoperability as result of more service-oriented demands exist. Reliable services and network performance act as technology requirements for more secure and reliable communication systems supporting end user and network requirements. Demands on data models integration are requirements to be considered during the design and implementation phases of any ICT system.

The emergence and wide-scale deployment of wireless access network technologies calls into question the viability of basing the future Internet on IP and TCP protocols that were never intended for use across highly unreliable and volatile wireless interfaces. Some, including the GENI NSF-funded initiative [1], to rebuild the Internet, argue that the future lies in layers of overlay networks that can meet various requirements whilst keeping a very simplistic, almost unmanaged, IP for the underlying Internet. Others initiatives such as Clean Slate program [2] Stanford University, and Architecture Design Project for New Generation Network [3] argue that the importance of wireless access networks requires a more fundamental redesign of the core Internet Protocols themselves.

We argue that service agnostic network design are no longer a way to achieve interactive solutions in terms of service composition and information sharing capabilities for heterogeneous infrastructure support. A narrow focus on designing optimal networking protocols in isolation is too limited. Instead, a more holistic and long-term view is required, in which networking issues are addressed in a manner that focuses on the supporting role various protocols play in delivering communications services that meet the rapidly changing needs of the communities of users for which the hour glass architecture model become in a critical infrastructure. 
In this paper service and network requirements [4][5][6][7][8][9] acts as inputs particularly on information interoperability and cross-domain information sharing controlling communication systems for the Future Internet. We support the idea of interoperable, extensible, reusable, common and manageable new Internet reference model is critical for Future Internet realization and deployment. The new Internet reference model must rely on the fact that high-level applications make use of diverse infrastructure representations and not use of resources directly. So when resources are not being required to support or deploy services they can be used in other tasks or services. As implementation challenge for controlling and harmonize this entire resource management requirements and architectural design issues the federation paradigm emerges as a tentative approach and optimal solution. We address challenges for a future Internet Architecture perspective using federation. We also provide, in a form of realistic implementations, research results and solutions addressing basics for federation.

Federated management scenarios are investigated [5][10] on what information enterprise application management systems can provide to allow the latter to more robustly and efficiently allocate network services.

This paper is organized as follows: Section II presents a brief review of the challenges about Future Internet architectures in terms of cross-domain interoperability. Section III presents the rationale about federation as crucial concept in the framework of this Future Internet research. Section IV presents a Federated Management Reference Model and its implications for networks and services. Section V describes what we consider as critical functional blocks for an Inter-disciplinary approach towards the specification of mechanisms for federated management. Section VI introduces End-to-End service management scenarios; we also investigate what information enterprise application management systems can provide to federated management systems allowing network and services allocation. Section VII presents the summary and outlook of this research. Finally some bibliography references supporting this research are included.

\section{Challenges for Future Internet Architectures}

This section focuses on inter-disciplinary approaches to specify data link and crossdomain interoperability to, collectively, constitute a reference model that can guide the realisation of future communications environments in the Future Internet [4][11][12] [13]. The Future Internet architecture must provide societal services and, in doing so, support and sustain interactions between various communities of users in straight relation with communication infrastructure mechanisms. Service-awareness [4] has many aspects to consider as challenges, including: delivery of content and service logic with consumers' involvement and control; fulfilment of business and other service characteristics such as Quality of Service (QoS) and Service Level Agreements (SLA); optimisation of the network resources during the service delivery; composition and decomposition on demand of control and network domains; interrelation and unification of the communication, storage, content and computation substrata.

Networking-awareness [4] challenges imply the consumer-facing and the resourcefacing services are aware of the properties, the requirements, and the state of the net- 
work environment, which enable services to self-adapt according the changes in the network context and environment. It also means that services are both executed and managed within network execution environments and that both the services and the network resources can be managed uniformly in an integrated way. Uniform management allows services and networks to harmonize their decisions and actions [14]. The design of both networks and services is moving forward to include higher levels of automation, and autonomicity, which includes self-management.

The optimization of resources [15][16][17] using federation in the Future Internet relies on classify and identify properly what resources need to be used, thus dynamically the service composition and service deployed can be executed by result of well known analysis on network and services.

\section{Rationale for Federation in the Future Internet}

Federation is relatively a new paradigm in communications, currently studied as the alternative to solve interoperability problems promoting scalability issues and exploring towards solving complexity when multiple applications/systems need to interact with a common goal. In this paper federation is handled as the mechanism used by communications management systems providing autonomic control loops.

In this section, the rationale for federated, autonomic management of communications services is addressed from the perspective of end-to-end applications and services in the Future Internet. Federation in the Future Internet envisions management systems (networks and services) made up of possibly heterogeneous components, each of which has some degree of local autonomy to realize business goals. Such business goals provide services that transcend legal and organizational boundaries in dynamic networks of consumers and providers. All the management systems with their own autonomy level contribute to satisfy more complex business goals, a single entity would not be able to achieve.

A visionary perspective for what federation can offer in communications systems and how federation contributes enabling information exchange has been described in previous works [18][19]. The intention in this paper is not to define what the Federation in future communications is, or which advantages it can offer either basics definition(s) in communications, but rather to provide a realistic approach in form of functional architecture, research results and implementation advances as well to show in kind how federation acts as feasible alternative towards solving interoperability problems in service and application management systems.

Future Internet environments consist of heterogeneous administrative domains, each providing a set of different services. In such complex environment, there is no single central authority; rather, each provider has at least one (and usually multiple) separate resources and/or services that must be shared and/or negotiated.

The term Federation in communications was discussed in a previous work [20] and currently many definitions have been proposed. We particularly follow a federated management definition as "A federation is a set of domains that are governed by either a single central authority or a set of distributed collaborating governing 
authorities in which each domain has a set of limited powers regarding their own local interests" because it fits better to management systems and due federation has two important implications nor considered in previous definitions i) federation must facilitates designing platforms without unnecessarily replicating functionalities, and ii) to achieve federation is necessary building inter-connected, inter-operating and/or inter-working platforms. Federation also implies that any virtual and /or real resource, which reside on another domain are managed correctly.

\section{$4 \quad$ Federated Management Activity in the Future Internet}

This section references theoretical foundation for the development of interdisciplinary Future Internet visions about a Federated Management and their implications for networks and services. These principles can be validated via direct industrial investment, and roll out real integrated test beds to trial new network and service infrastructures.

In future Internet end user, service, application and network requirements act as guidelines to identify study and clarify part of complex requirements. The relationships between Network Virtualisation and Federation [16][21][22][23] and the relationship between Service virtualisation (service clouds) and federation [17] are the support of a new world of solutions defining the Future Internet.

Next generation networks and services [3][4][24] can not be conceived without systems acting and reacting in a dynamic form to the changes in its surrounding (context-awareness, data link and information interoperability), even more the systems must be able for self-managing considering end-user requirements and acting in autonomous forms offering added value services (Autonomics) [6][7][25] where traditional definitions describing self-management emerged. However, most of them are based on very-high level human directives, rather than fully or partially automatic low-level management operations. While many aspects of the network will be selfmanaged based on high-level policies, the aggregation and subsequent understanding of monitoring/fault data is a problem that has not yet been completely solved here is where federation take place and acquire importance.

\subsection{Federated Autonomic Management Reference Model}

Federated refers to the ability of a system to enable network and service management as result of threading negotiations for evolving value chains composed of providers and/or consumers [14]. Autonomic reflects the ability of such systems to be aware of both themselves and their environment, so that they can self-govern their behaviour within the constraints of the business goals that they collectively seek to achieve. Management refers to the ability of such systems not just to configure, monitor and control a network element or service, but also to optimize the administration of lifecycle aspects of that resource(s) or service(s) in a programmable way. This enables end-users to take a more proactive role managing their quality of experience in a semantic way. In the depicted representation for the federated autonomic management 
reference model shown in the Figure 1 service and network domains must interact to exchange relevant information facilitating services and network operations. These cross-domain interactions demand certain level of abstraction to deal with mapping requirements from different information and data domains. This higher level of abstraction enables business and service foundations to be met by the network, and emphasizes offering federated services in a portable manner that is independent of the utilized networks. The objective is to effectively deliver and manage end-to-end communications services over an interconnected, but heterogeneous infrastructure and establishes communication foundations.

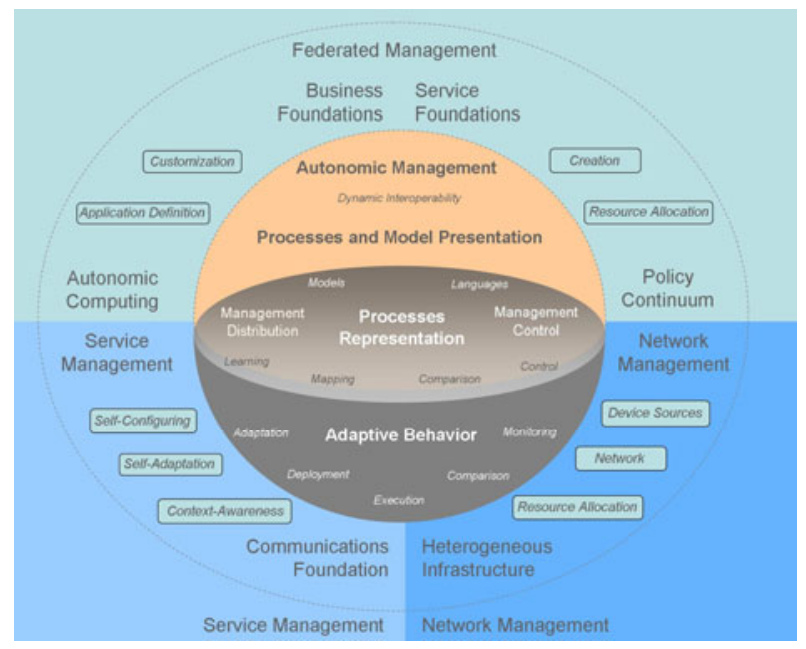

Fig. 1. Federated Autonomic Management Reference Representation

A greater degree of coordination and cooperation is required between communication resources, the software that manages them, and the actors who direct such management. In federation management end-to-end communication services involve configuring service and network resources in accordance to the policies of the actors involved in the management process. An autonomic management system provides automatic feedback to progressively improve management policies as service usage and resource utilization change. A goal of autonomic systems is to provide rich usage data to guide rapid service innovation.

Concepts related to Federation such as Management Distribution, Management Control and process representation are clear on their implications to the network management, however up to date there are no clear implications around what federation offers in communications either what federation to the next generation networks and in the Future internet design with service systems using heterogeneous network technologies imply. A clear scenario where federation is being identified as useful mechanism is the Internet service provisioning, in today's Internet it is observed the growing trend for services to be both provided and consumed by loosely coupled value networks of consumers, providers and combined consumer and providers. These consumer valued networks acting "ideally" as independent self-management entities must combine efforts 
to offer "common" and "agreed" services even with many technological restrictions and conflicts blocking such activity. A set of scenarios is introduced in a following section describing federation in more detail.

\subsection{Federated Management Service Life Cycle}

Management and configuration of large-scale and highly distributed and dynamic enterprise and networks applications [26] is everyday increasingly in complexity. In the current Internet typical large enterprise systems contain thousands of physically distributed software components that communicate across different networks [27] to satisfy end-to-end services client requests. Given the possibility of multiple network connection points for the components cooperating to serve a request (e.g., the components may be deployed in different data centres), and the diversity on service demand and network operating conditions, it is very difficult avoid conflicts [14][20][28] between different monitoring and management systems to provide effective end-toend applications managing the network.

The Figure 2 depicts the federated autonomic reference model service life cycle for the Future Internet. We are exploring how the definition and contractual agreements between different enterprises (1.Definition) establish the process for monitoring (2.Observation) and also identify particular management data at application, service, middleware and hardware levels (3.Analysis) that can be gathered, processed, aggregated and correlated (4.Mapping) to provide knowledge that will support management operations of large enterprise applications (5.Federated Agreements) and the network services they require (6.Federated Regulations). We support the idea that monitoring data at the network and application level can be used to generate knowledge that can be used to support enterprise application management in a form of control loops in the information; a feature necessary in the Future Internet service provisioning process (7.Federated Decisions). Thus infrastructure can be re-configurable and adaptive to business goals based on information changes (8.Action).

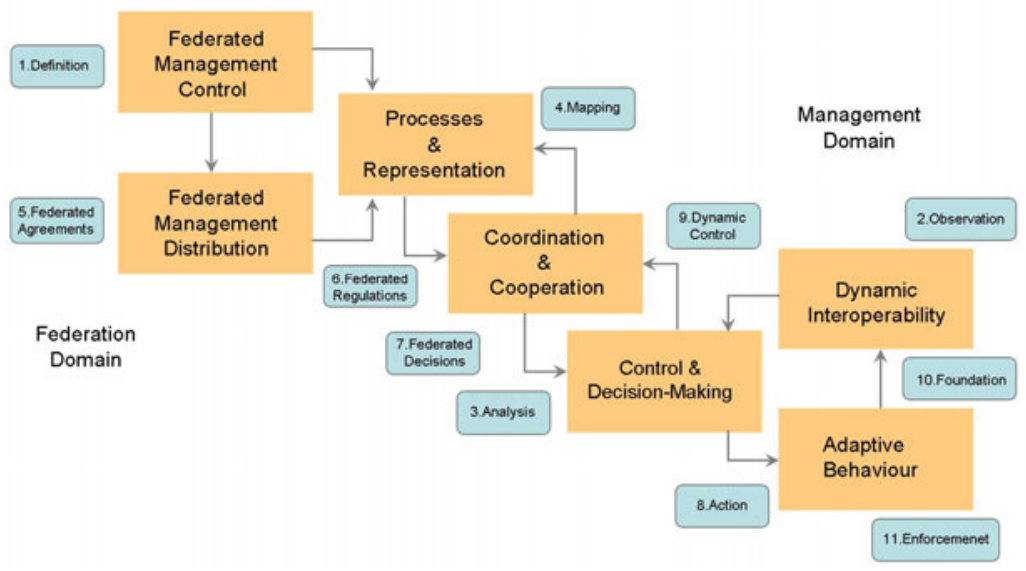

Fig. 2. Federated Management Life Cycle Reference Model 
We also consider appropriate ways on how information from enterprise applications and from management systems can be provided to federate management systems allowing to more robustly and efficiently be processed to generate adaptive changes in the infrastructure (9.Dynamic Control). Appropriate means of normalising, interpreting, sharing and visualising this information as knowledge (10.Foundations) thus allocate new federated network services (11.Enforcement).

In a federated system the interaction between domains and the operations in between represent a form of high-level control to perform the negotiations and regulations to achieve the compromises pertaining to a federation and mainly resolve negotiations (represented as transition processes normally) not considered between individual or autonomous self-management domains. The transitions processes are not a refinement or finite process itself rather than that a transition represents information transformed into knowledge and their respectively representation. The operations and processes management in a federated architecture must support a finite goal to define, control, and coordinate service and network management conditions as much as possible from an application management high level control view, the so called federation. The federated functional architecture and its logic operations are depicted in Figure 2 and described more in detail here after.

- Dynamic Interoperability - Autonomic functionality, which can be result of negotiations between management components, devices or systems by using a same language or information model, non formal representation is necessary.

- Adaptive Behaviour - Autonomic and inherent functionality assigned to the components can be managed by federated conditions and regulations, which communicate with other non-autonomic components, thus virtual and/or real resources can be expand and contract the network infrastructure.

- Control and Decision-Making - The Functionality of a component(s) and system(s) to conduct its own affairs based on inputs considered as conditions and define outputs considered as actions.

- Coordination \& Cooperation - Functionality associated to promote and resolve high level representation and mapping of data and information. Negotiations in form of data representation between different data and information models by components in the system(s) are associated to this feature.

- Management Control - Administration functionality for the establishment of cross-domain regulations considering service and network regulations and requirements as negotiations.

- Management Distribution - Organizational functionality for the adoption and enforcement of cross-domain regulations as result of service and network requirements.

- Process \& Representation - Autonomic functionality assigned to components or systems, orchestrating the behaviour(s) in the components of managed systems. 


\section{Federated Management Architecture}

This section describes designing principles for inter-domain federated management architectures in the Future Internet. These designs about architecture for the federated reference model by functional blocks addresses the specification of mechanisms including models, algorithms, processes, methodologies and architectures. The functional architecture collectively constitute, in terms of implementation efforts, framework(s), toolkit(s) and components that can guide the realisation of federated communications environments to effectively provide complex services (interoperable boundaries) and, in doing so, support and sustain service offering between various communities of users (heterogeneous data $\&$ infrastructure).

The federated architecture must be enabled for ensuring the information is available allowing useful transfer of knowledge (information interoperability) across multiple interfaces. It is likely that adaptive monitoring is used to optimise the efficiency of the federated process. A specific set of service applications, domain independent, and configurations for managing services and networks are used to ensure transference of results to other systems as result of sensitivity analysis. Simulation studies and analytical work is being conducted to back up further experimental results.

Designing a federated platform implies the combination of semantic descriptions and both holistic service and management information. When using semantics the interaction between systems named interactive entities is to reduce the reliance on technological dependencies for services support and increasing the interoperability between heterogeneous service and network management systems. A federated autonomic architecture must supports such interactions offering a full service lifecycle control by using federated autonomic mechanisms where relations and interactions for unified management operations are based on the use of formal mechanisms between different domains. This interaction relies on supporting end-user interface components ensuring high level management systems information exchange.

\subsection{Federated Management Enforcement Process}

The purpose of federation in autonomic is to manage complexity and heterogeneity. In a federated autonomic network, time-consuming manual tasks are mostly or completely automated; and decisions delegated, this dramatically reduces manuallyinduced configuration errors, and hence lowers operational expenditures when decision needs to be implemented. By representing high level business requirements in a formal manner, information and data can be integrated, and the power of machinebased learning and reasoning can be more fully exploited. Autonomic control loops and its formalisms [29][30], such as FOCALE [25] and AutoI [21][23] translate data from a device-specific form to a device- and technology-neutral form to facilitate its integration with other types of information. The key difference in the autonomic control loop, compared with a non-autonomic control loop, is the use of semantic information to guide the decision-making process. The key enabling federation is the process of semantic integration that can create associations and relationships among enti- 
ties specified in different processes and formal representations acting as foundations into the system.

The following paragraphs are concentrated to describe the key logic domains which the federated autonomic architecture concentrates and which have been identified as main interest research topics around federation. The interactions between the logic areas are represented by cursives. As shown in figure 3. The design of a federated autonomic architecture enables transitions from high-level goals (as codified by service rules for example) to low-level as network policies. In a federated autonomic architecture, information is used to relate knowledge, rather than only map data, at different abstractions and domain levels co-relating independent events each other in a federated way. We envisage federation of networks, network management systems and service management applications at three levels of abstraction.

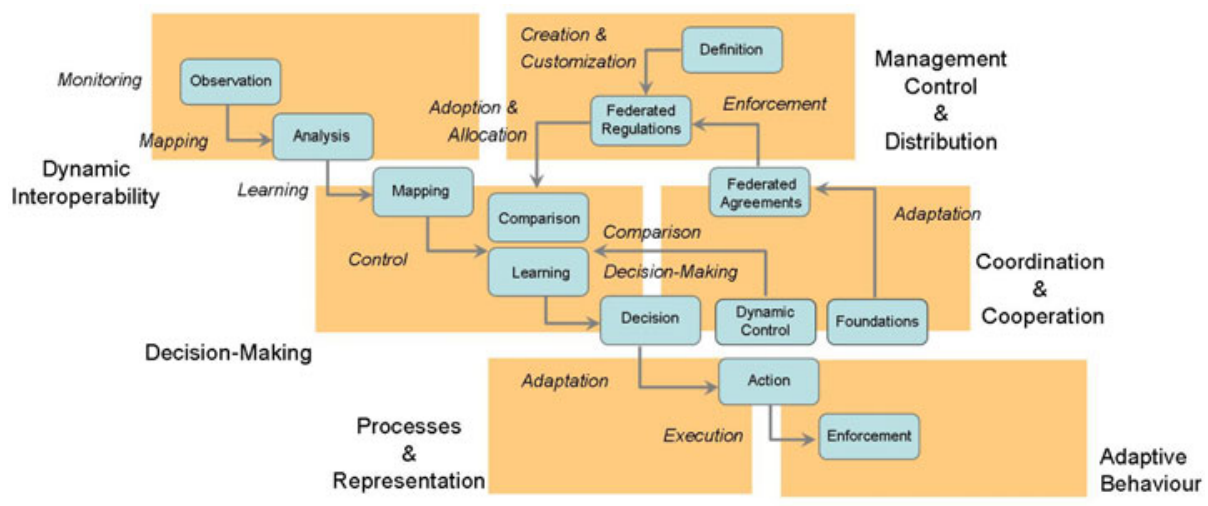

Fig. 3. Federated Management Enforcement Process

At the lowest level adaptive behaviour and processes \& representation, at this level is the heterogeneous infrastructure where networks and devices coordinate selfmanagement operations. Management systems should support self-management by local resources in a given domain ensuring that this self-managed behaviour is coordinated across management boundaries. In the top level, Management Control \& Distribution and Dynamic Interoperability termed federation domain, the vision of federated autonomic management for end-to-end communications services orchestrate federated service management where management systems should semantically interoperate to support evolving value chains and the end-to-end delivery of services.

At the middle level, coordination \& cooperation and decision-making where multiple management domains (service, application and networking) should interact and interoperate for configuring network resources in a consistent way, following with federated business goals, but in a manner that is consistent with configuration activity in neighbouring network domains participating in a value network. The federated autonomic architecture proposed can be seen itself as a federated system where exist regulations defining the performance or the behaviour in the heterogeneous infrastructure, such regulations are transformed using adaptations processes and formal representation to express and deploy the conditions comprised and described by the man- 
agement distribution. Such regulations must be deployed with no further consideration from other systems or sub-systems; this feature is used when conflicts and negotiations need to be performed in the federation space.

In the federated architecture proposed the management control deal with federated agreements necessaries to satisfy in one hand the enterprise requirements and in the other hand the management system requirement as result of events coming from the heterogeneous infrastructure. The events are expressed in a form of coordination and cooperation functions, which have origins in mapping events between the diverse enterprise processes and the heterogeneous infrastructure. The federation also acts as mediator between autonomic and/or non-autonomic management sub-systems when controlling each one independently their behaviour and a higher level mechanism is necessary to act as a one in pursuing a common goal, in an heterogeneous service deployment and support for example.

\section{End-to-End Federated Service Management Scenarios}

In this scenarios description section conversely, we also provide research results about what information enterprise application management systems can provide to federate management systems to allow the latter to more robustly and efficiently allocate network services. Brief scenario descriptions illustrate the possible challenges are necessaries to tackle around the term federation and particularly on federated systems and federated management applications.

\subsection{Federation of Wireless Networks Scenario}

generates more demand on management systems to be implemented satisfying diversity, capacity and service demand. Given the fact that in urban areas (shopping centres, apartment buildings, offices) generates more demand in deploying wireless 802.11-based mesh networks this expansion will be a patchwork of mesh networks; challenges arise relating to how services can be efficiently delivered over these overlapping infrastructures. Challenges in wireless mesh networks relate to both resource management within the network infrastructure itself and the way in which management systems of individual network domains can federate dynamically to support endto-end delivery of services to end-users. Furthermore, there are challenges relating to securing the delivery of services across (possible multiple) wireless mesh infrastructure domains.

This research scenario opens work mainly for focusing on the specifics of resource management within multi-provider mesh networks by using federation principles (federated management). The exact nature of the mesh networks to be used in multiprovider networks is not yet clear. However, from a management system perspective, the scope of this scenario rely in the fact on how the use of semantic models capturing knowledge relating to security functionality and the use of policy rules to control endto-end configuration of this functionality can provide a basis for the support flexible trust distributed management across wireless meshes, (federated deployment). 


\subsection{Federation of Network and Enterprise Management Systems}

Typical large enterprise systems contain thousands of physically distributed software components that communicate across different networks to satisfy client requests. Management and configuration is increasingly complex at both the network and enterprise application levels. The complex nature of user requests can result in numerous traffic flows within the networks that can not be correlated with each other, thus which it ideally should be treated in a federated fashion by the networks. Challenges in this scenario relies on how monitoring at the network level can provide knowledge that will enable enterprise application management systems to reconfigure software components to better adapt applications to prevailing network conditions. This reconfiguration may, for example, involve redeployment of application components in different locations by using different infrastructures (federation) in order to alleviate congestion detected within particular parts of the network. Conversely, the information enterprise application management systems can provide to network management systems allowing a more efficient and cost-effectively manage traffic flows relating to complex transactions (federated management).

This scenario carry on work for developing federated monitoring techniques that can be applied to record and analyse information and trends in both network management systems and enterprise application management systems, in a manner such that a coherent view of the communication needs and profile of different transaction types can be built. Enterprise application management systems must be specified to provide relevant application descriptions and behaviours (e.g., traffic profiles and QoS levels) to network management system allowing shared knowledge to be optimally used (federation) in network traffic management processes.

\subsection{Federation of Customer Value Networks Scenario}

Network service usage is increasingly billed as flat-rate Internet access. Within the "Web 2.0" development, online value is expanding from searching and e-consumerism applications, to participative applications including blogs, wikis, online social networks, RSS feeds, Instant Messaging, P2P applications, online gaming and increasingly pervasive VoIP applications. Particularly as users have been empowered to mix and match applications to create customised functionality (e.g. mash-ups). Similarly, at a business or organisational level, the knowledge sector of modern economies is increasingly focussed on value networks rather than on value chains. Value networks offer benefits that are more intangible (e.g. the value of professional contact networks). Value networks share with Web 2.0 application users a concern with value of interacting effectively with rest of the network community (federation).

In highly active value networks, the cost of interrupted interactions may be perceived as high; however, there is little visibility of the root responsible source of interaction breakdowns between the various communication services providers, application service hosts, or value network members themselves. Due to this lack of visibility, value networks have very limited avenues for taking remedial or preventative actions, such as recommending different network or service providers to their mem- 
bers. Value networks of customers can only properly be served by federated service providers, henceforth termed Service Provider Federation (SPF).

\subsection{Federation of Home Area Networks Services and Applications}

An emerging trend in communications networks is the growing complexity and heterogeneity of the "outer edge" domain - the point of attachment of Home Area Networks (HANs) and other restricted area networks to various access networks. Challenges on this scenario must address management of outer edge devices, such as femto base stations, home gateways, set-top boxes and of networks thereof. Today this task is provided on an piecemeal basis, with different devices having a wide range of management functionality, which is often proprietary or, at best, conforms one of a range of competing standards. Furthermore, management operations must be performed by end-users. Achieving this requires increased degrees of integration between telecommunications network management systems and devices. In particular, it is important to develop methods (management functions) through which network management systems can assume responsibility for appropriate configuration of HAN devices. A significant challenge in this regard is that as the diversity and capabilities of HAN devices increases.

To address these scenario requirements the use of distributed event processing and correlation techniques that can process relevant data in a timely and decentralised manner and relay it as appropriate to management federated making functions are necessaries to investigate (federation). This scenario discloses on aspects about federation and integrated management of outer edge network environments; delegation of management authority to network management systems and decentralised assurance of service delivery in a home area are important too.

\section{$7 \quad$ Summary and Outlook}

In the future Internet new designs ideas of Federated Management in Future Internet Architectures must consider high demands of information interoperability to satisfy service composition requirements being controlled by diverse, heterogeneous systems make more complex perform system management operations. The federated autonomic reference model approach introduced in this paper as a design practice for Future Internet architectures emerges as an alternative to address this complex problem in the Future Internet of networks and services.

We have studied how federation brings support for realisation on the investigated solution(s) for information interoperability and cross-domain information sharing controlling communication systems in the Future Internet. Additional issues such as service representation and networks information can facilitate service composition and management processes. Remaining research challenges regarding information model extensibility and information dissemination exist and would be conducted to conclude implementations, experiments composing services in some of the scenarios described in this paper. 


\subsection{Research Outputs as Rationale for Federation}

- Techniques and agreements for composition/decomposition of federated control frameworks for physical and virtual resources and systems

- Techniques and mechanisms for controlling workflow for all systems across federated domains, ensuring bootstrapping, initialisation, dynamic reconfiguration, adaptation and contextualisation, optimisation, organisation, and closing down of service and network components.

- Mechanisms for dynamic deployment on-the-fly of new management functionality without running interruption of any systems across multiple and federated domains.

- Algorithms and processes to allow federation in enterprise application systems to visualize software components, functionality and performance.

- Techniques for analysis, filtering, detection and comprehension of monitoring data in federated enterprise and networks.

- Algorithms and processes to allow federated application management systems reconfigure or redeploy software components realizing autonomic application functionality.

- Guidelines and exemplars for the exchange of relevant knowledge between network and enterprise application management systems.

This paper makes references to design foundations for the development of federated autonomic management in architectures in the Future Internet. Scenarios has been shortlisted to identify challenges and provide research results about what information enterprise application management systems can provide to federate management systems by using an interoperability of information as final objective.

Acknowledgements. The work introduced in this paper is a contribution to SFI FAME-SRC (Federated, Autonomic Management of End-to-End Communications Services - Scientific Research Cluster). Activities are partially funded by Science Foundation Ireland (SFI) via grant 08/SRC/I1403 FAME-SRC (Federated, Autonomic Management of End-to-End Communications Services - Scientific Research Cluster) and by the UniverSELF EU project [31], grant agreement $n^{\circ} 257513$, partially funded by the Commission of the European Union.

Open Access. This article is distributed under the terms of the Creative Commons Attribution Noncommercial License which permits any noncommercial use, distribution, and reproduction in any medium, provided the original author(s) and source are credited.

\section{References}

1. NSF-funded initiative to rebuild the Internet (Online: Oct. 2010), http: / /www.geni.net/

2. Clean Slate Program, Stanford University (Online: Nov. 2010), http: //cleanslate.stanford.edu/

3. Architecture Design Project for New Generation Network (Online: Oct. 2010), http://akari-project.nict.go.jp/eng/index2.htm 
4. Galis, A., et al.: Management and Service-aware Networking Architectures (MANA) for Future Internet Position Paper: System Functions, Capabilities and Requirements (Invited paper). In: IEEE 2009 Fourth International Conference on Communications and Networking in China (ChinaCom09) 26-28 August, Xi'an, China (2009)

5. Clark, D., et al.: NewArch: Future Generation Internet Architecture., NewArch Final Technical Report, http: / /www.isi.edu/newarch/

6. van der Meer, S., Davy, A., Davy, S., Carroll, R., Jennings, B., Strassner, J.: Autonomic Networking: Prototype Implementation of the Policy Continuum. In: Proc. of 1st IEEE International Workshop on Broadband Convergence Networks (BcN 2006), in conjunction with NOMS 2006, Canada, April 7, 2006, pp. 163-172. IEEE Press, Los Alamitos (2006)

7. van der Meer, S., Fleck II, J.J., Huddleston, M., Raymer, D., Strassner, J., Donnelly, W.: Technology Neutral Principles and Concepts for Autonomic Networking. In: Advanced Autonomic Networking and Communication, Birkhäuser, Basel (2008)

8. Raymer, D., van der Meer, S., Strassner, J.: From Autonomic Computing to Autonomic Networking: an Architectural Perspective. In: Proc. of 5th IEEE Workshop on Engineering of Autonomic and Autonomous Systems (EASe 2008), Co-located with ECBS, Belfast, United Kingdom, March 31-April 4 (2008)

9. Jennings, B., van der Meer, S., Balasubramaniam, S., Botvich, D., Foghlú, M.Ó., Donnelly, W., Strassner, J.: Towards Autonomic Management of Communications Networks. IEEE Comms Magazine, IEEE 45(10), 112-121 (2007)

10. Blumenthal, M., Clark, D.: Rethinking the design of the Internet: the end to end arguments vs. the brave new world. ACM Transactions on Internet Technology 1(1) (2001)

11. Subharthi, P., Jianli, P., Raj, J.: Architectures for the Future Networks and the Next Generation Internet: A Survey. Computer Communications (July 2010), 63 pp., http: / /www1.cse.wustl.edu/ jain/papers/ftp/i3survey.pdf

12. Curran, K., Mulvenna, M., Galis, A., Nugent, C.: Challenges and Research Directions in Autonomic Communications. International Journal of Internet Protocol Technology (IJIPT) 2(1) (2006)

13. Rubio-Loyola, J., Astorga, A., Serrat, J., Chai, W.K., Mamatas, L., Galis, A., Clayman, S., Cheniour, A., Lefevre, L., Fischer, A., Paler, A., Al-Hazmi, Y., de Meer, H.: Platforms and Software Systems for an Autonomic Internet. In: IEEE Globecom 2010, Miami, USA, 610 December (2010)

14. Jennings, B., Brennan, R., van der Meer, S., Lewis, D., et al.: Challenges for Federated, Autonomic Network Management in the Future Internet. In: ManFI workshop, June, NY, USA (2009)

15. Strassner, J.C., Foghlú, M.Ó., Donnelly, W., Serrat, J., Agoulmine, N.: Review of knowledge engineering requirements for semantic reasoning in autonomic networks. In: Ma, Y., Choi, D., Ata, S. (eds.) APNOMS 2008. LNCS, vol. 5297, pp. 146-155. Springer, Heidelberg (2008)

16. Strassner, J., Foghlú, M.Ó., Donnelly, W., Agoulmine, N.: Beyond the Knowledge Plane: An Inference Plane to Support the Next Generation Internet. In: IEEE GIIS 2007, 2-6 July (2007)

17. Galis, A., Denazis, S., Brou, C., Klein, C.: Programmable Networks for IP Service Deployment. Artech House Books, London (2004)

18. Serrano, M., Strassner, J., Foghlú, M.Ó.: A Formal Approach for the Inference Plane Supporting Integrated Management Tasks in the Future Internet. In: 1st IFIP/IEEE ManFI Intl Workshop, In conjunction 11th IEEE IM2009, Long Island, NY, USA, June 2009, IEEE Computer Society Press, Los Alamitos (2009)

19. Brennan, R., Feeney, K., Keeney, J., O’Sullivan, D., Fleck II, J., Foley, S., van der Meer, S.: Multi-Domain IT Architectures for Next Generation Communications Service Providers. IEEE Communications Magazine 48, 110-117 (2010) 
20. Serrano, J.M., van der Meer, S., Holum, V., Murphy, J., Strassner, J.: Federation, A Matter of Autonomic Management in the Future internet. In: IEEE/IFIP Network Operations \& Management Symposium, NOMS 2010, Osaka, Japan, 19-23 April (2010)

21. Bassi., A., Denazis., S., Galis., A., Fahy., C., Serrano., M., Serrat, J.: Autonomic Internet: A Perspective for Future Internet Services Based on Autonomic Principles. In: 2007 IEEE Management Week - ManWeek 2007 2nd IEEE MACE 2007 Workshop, San José, CA, USA, 29 Oct. -2 Nov (2007)

22. Rochwerger, B., et al.: An Architecture for Federated Cloud Computing. In: Cloud Computing: Principles and Paradigms, Wiley, ISBN: 0470887990 (April 2011)

23. Galis, A., et al.: Management Architecture and Systems for Future Internet Networks. In: Towards the Future Internet - A European Research Perspective, p. 350. IOS Press, Amsterdam (2009)

24. Feldmann, A.: Internet clean-slate design: what and why? ACM SIGCOM Computer Communication Review 37(3) (2007)

25. Strassner, J., Agoulmine, N., Lehtihet, E.: FOCALE - A Novel Autonomic Networking Architecture. ITSSA Journal 3(1), 64-79 (2007)

26. Foley, S.N., Zhou, H.: Authorisation Subterfuge by Delegation in Decentralised Networks. In: Proc. of the 13th International Security Protocols Workshop, Cambridge, UK (April 2005)

27. Jennings, B., et al.: Towards Autonomic Management of Communications Networks. IEEE Comms Magazine 45(10), 112-121 (2007)

28. Strassner, J.: Autonomic Networks and Systems: Theory and Practice. In: NOMS 2008 Tutorial, Brasil (April 2008)

29. Strassner, J., Kephart, J.: Autonomic Networks and Systems: Theory and Practice. In: NOMS 2006 Tutorial (Apr 2006)

30. Serrano, J.M.: Management and Context Integration Based on Ontologies for Pervasive Service Operations in Autonomic Communication Systems., PhD Thesis, UPC (2008)

31. UniverSELF Project (January 2011), http: //www. univerself-project.eu/ 